\title{
ANALYSIS OF DENOISING INTERPRETATION OF REMOTE SENSING IMAGE BASED ON ICA-WAVELET TRANSFORM
}

\author{
SUN Lin ${ }^{1}$, GAN Xiaosu ${ }^{2}$ \\ ${ }^{1}$ Beijing HeadSpring Technology Co.Ltd, BEIJING, CHINA \\ ${ }^{2}$ China Transport Telecommunications and Information Center, BEIJING, CHINA
}

KEY WORDS: ICA-Wavelet analysis, image quality inspection, image interpretation

\begin{abstract}
:
The noise will blur the key information of the remote sensing image, such as edge texture and important feature information, which will result in the loss of key information contained in the remote sensing image, resulting in the degradation of the overall quality of the image, which will bring difficulties to the interpretation work. Therefore, in order to obtain higher precision, signal-to-noise ratio and improve the quality of remote sensing image, denoising the remote sensing image containing noise is a crucial step and processing step for image remote sensing image application.

In this paper, the ICA wavelet analysis algorithm is applied to the application of real-time remote sensing image denoising. A series of pre-processing procedures such as control point correction, image fusion and image mosaic are carried out on the Asian sub-level remote sensing image, and the signal-to-noise ratio of the remote sensing image is adopted. (SNR/dB) and mean square error (RMSE) verify the image quality after denoising.
\end{abstract}

\section{INTRODUCTION}

How to remove noise in remote sensing images is an important topic in the field of remote sensing image information processing. It has existed since the birth of remote sensing technology, and it has become more and more prominent with the development of remote sensing technology. In order to solve this problem, many scholars have proposed a variety of noise reduction methods from the perspective of remote sensing image characteristics, noise statistical characteristics, spectrum distribution law. But the problem that always precedes the academic interface is how to protect the details of the image as much as possible during the noise reduction process.

With the massive increase of high-resolution remote sensing data in recent years, how to accurately and efficiently distinguish between high-frequency signals and high-frequency noise is an important problem to be solved for high-resolution image processing.

\section{KEY TECHNOLOGY}

Gradually deeper use of wavelet denoising, on the basis of conventional wavelet denoising, the noise reduction effect of independent component analysis wavelet is cited.

\subsection{Wavelet noise reduction}

The model chosen for the problem of noise denoising is: $s(i)=f(i)+e(i), i=0,1, \ldots, n-1$

Here, $f(i) 、 s(i) 、 e(i)$ representing the desired image, the observed noisy image, and the noise, respectively. e stands for noise variance denoising. Simply speaking, the original image $S(i)$ is restored in the noisy image. At the same time, the image $S(i)$ needs to maintain its original features, and the mean square error is optimized. Simply speaking, it is a set of orthogonal $B=\left\{g_{m}\right\},(0 \leq m \leq N)$ The base is decomposed in this way $s(i)=f(i)+e(i)$ and then you can get:

$\left\langle s(i), g_{m}\right\rangle=\left\langle f(i), g_{m}\right\rangle+\left\langle e(i), g_{m}\right\rangle$

In the time-frequency domain, the wavelet function has a relatively good locality, and the variable-scale characteristics of the wavelet function can make the wavelet transform have the "concentration" ability in the process of processing the signal, and can relatively better the local characteristics of the signal. Show it out. Therefore, the basic principle of wavelet transform denoising is that, based on the local structural features, the Lipschilz index of noise and signal can exhibit a certain singularity, and then it is properly processed by wavelet coefficients.

\subsection{ICA-wavelet noise reduction}

Incdpendent Component Analysis(ICA)is an analytical method proposed when studying the separation of blind sources. In the actual use of the noise analysis model, the phenomenon that the observed signal and the noise appear at the same time can always be found, so the influence of the noise should be added in the design. Models with noise considerations are very realistic, and the ICA expression for noise is:

$$
x(t)=A s(t)+n(t)
$$

A is a kind of mixing matrix, $a_{j}$ which is the basis vector of the mixing matrix. The source signal $\mathrm{s}(\mathrm{t})$ and the mixing matrix $\mathrm{A}$ are all unknown, and $x(t)$ can be observed after signal mixing. 
In order to achieve a simplified structure and a simple operation, image processing is often performed on the ICA algorithm, and the image processing result is firstly whitened.

(1) Finally, the desired analysis results are obtained by simple calculation. If the actual situation is far from the zero-mean situation, it needs to be improved by means of differentiation. The implementation of centralization is as follows:

$$
x \leftarrow x^{\prime}-E\left\{x^{\prime}\right\}
$$

At the same time, after the process is completed, the independent component is changed to zero mean:

$$
\mathrm{E}\{\mathrm{s}\}=\mathrm{A}^{-1} E\{x\}
$$

(2) Using the whitened matrix, a simpler matrix form can be obtained, with a simple data structure and a highly efficient algorithm. Since the correlation between the variables after whitening completely disappears, the results can be analyzed using the principal quantities.

Since the correlation between variables after the whitening process is completely eliminated, it is often confused with irrelevance. Whitening needs to meet the following requirements:

$$
m_{x}=0, R_{x}=C_{x}=I
$$

The process of whitening is the multiplication operation between the vector and the matrix, which can be decomposed by introducing a covariance matrix:

$$
E\left\{x x^{T}\right\}=E D E^{T}
$$

The average value of data after whitening is 0 , and there is no correlation between all matrices after whitening. If you find the covariance matrix of the whitened matrix, you can get the correct result.

$$
\begin{aligned}
& C=\left[\begin{array}{cccc}
c_{11} & c_{12} & \ldots & c_{1 n} \\
c_{21} & c_{22} & \ldots & c_{2 n} \\
\ldots & \ldots & \ldots & \ldots \\
c_{n 1} & c_{n 2} & \ldots & c_{n n}
\end{array}\right] \\
& C_{i j}=\operatorname{cov}\left[x_{i} x_{j}\right]=E\left\{\left[x_{i}-E\left(x_{i}\right)\right]\left[x_{j}-E\left(x_{j}\right)\right]\right\}
\end{aligned}
$$

The calculation result of the equation after the centralization should be 1 . After the whitening process, the correlation of the data disappears completely, so $c_{i j}=0,(i \neq j)$ 。

\section{ICA-WAVELET NOISE REDUCTION METHOD}

\subsection{ICA-wavelet analysis model with noisy signals}

The noise in the image is regarded as additive white Gaussian noise, then the image system model is:

$$
x=A s+n \text {, }
$$

Among them, $s=\left[s_{1}(t), s_{2}(t), \ldots, s_{n}(t)\right]^{T}$ represents an unknown image source signal, where $\mathrm{A}$ is represented by an $n \times m$ unknown mixing matrix, $n=\left[n_{1}, n_{2}, \ldots, n_{m}(t)\right]^{T}$ representing additive white Gaussian noise.

For any image observation signal $x_{i}(t)$, it is linearized $s_{i}(t)$ by $n$ unknown image source signals. At this point, all we have to do is to find each unknown image source $s_{i}(t)$ from any of the observed signals $x_{i}(t)$.
For the above model, the basic process of solving with independent component analysis is as follows: $x$ is an image observation signal with Gaussian white noise, and $\mathrm{A}$ is an unknown mixing matrix, which is satisfied $y(t)=B x(t)$ by solving a mixing matrix $\mathrm{B}$, so that the signal $\mathrm{y}(t)$ is approximated to the source signal $s(t)$. At this time, it is necessary to ensure that the components $\mathrm{y}(t)$ are independent of each other.

\subsection{Wavelet transform and ICA separation}

ICA theory often ignores noise when performing signal processing, which makes ICA less effective when applied to noise signal separation. Wavelet transform is theoretically a data analysis method with multi-resolution analysis characteristics. It can decompose the noisy signal in multiple layers, so as to obtain the profile and some details of the noisy signal transformation, and then carry out some details. Threshold processing can suppress the noise; then the inverse details of the processing details and the profile can be used to obtain the denoising signal.

\section{ICA-WAVELET NOISE REDUCTION METHOD APPLICATION}

\subsection{Experimental area}

Shunyi, affiliated to Beijing, is located in the northeast of Beijing, 30 kilometers away from the urban area, bordering Huairou District and Miyun District in the north, Pinggu District in the east, and Tongzhou District in the south, Sanhe City in Hebei Province, and Changping District in the west. Chaoyang District is separated by Wenyu River. Between N40 - $00^{\prime}-40^{\circ} 18^{\prime}$, E116 $28^{\circ}-116^{\circ} 58^{\prime}$, the territory is 45 kilometers long from east to west, 30 kilometers wide from north to south, with a total area of 1021 square kilometers.

The terrain of Shunyi District is high in the north and low in the south. The northeast border barrier is Yanshan. The plains in the territory are caused by river floods carrying sediments. The surface deposits are mainly sand and sub-sand, accounting for $95.7 \%$ of the area. The highest point in the northern mountainous region is 637 meters above sea level, and the lowest point in the territory is 24 meters above sea level with an average elevation of 35 meters.

\subsection{Remote sensing image data}

The data was selected from the Beijing No.2 sub-meter highresolution satellite imagery PMS data as experimental sample data. The image data was selected in April 2019 with a spatial resolution of $0.8 \mathrm{~m}$, the image capturing side swing angle $<15^{\circ}$ , and the data width was $24 \mathrm{~km} \times 24 \mathrm{~km}$. Beijing No. 2 satellite sensor parameters are as follows:

\begin{tabular}{|c|c|}
\hline Band & Range $(\mathrm{nm})$ \\
\hline Blue & $440-510$ \\
\hline Green & $510-590$ \\
\hline Red & $600-670$ \\
\hline Panchromatic & $450-650$ \\
\hline Near infrared & $760-910$ \\
\hline
\end{tabular}




\subsection{Remote sensing image preprocessing}

(1) Control point acquisition

The imaging process of the remote sensing image sensor itself is very complicated. The error, atmospheric radiation and solar elevation angle are different. There are errors in measurement error and poor terrain conditions, which will make the results obtained in the measurement process inaccurate. Large measurement error. Using PIEOthro software, the obvious control points of the ground objects are collected, and the number of images collected per scene is not less than 8000 .

(2) Same name image acquisition

Similar to the control point acquisition method, the control points of the same name are collected on the adjacent two scenes and above, and the image points of the same name are evenly distributed, and the number of collection points is not less than 13 /square kilometers.

(3) Orthorectification correction

In the CGCS2000 coordinate system, the acquired control points are orthorectified with the same-named image points. Corrected using Spline's registration method.

(4) Image fusion

The calibrated panchromatic image is blended with the dominant band of the multi-spectral image, retaining the color information of the high-resolution and multi-spectral images of the panchromatic image.

(5) Image mosaic

In order to meet the full coverage of the image in Shunyi District, the experiment used a total of 7 scenes of Beijing No. 2 image for pretreatment. The fused 7-frame remote sensing image is mosaic-stitched.

(6) Image cropping

Since the range of the imaged image is too large, the image is cropped according to the administrative scope of the Shunyi District.

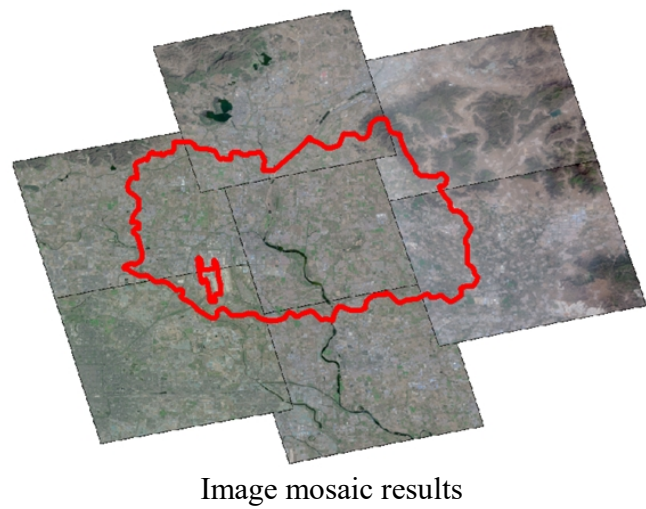

(7) Band preference

After the integration of the PMS effects, four bands are obtained. There is a big difference between the content that can be reflected by each band and the characteristics of the region's organisms. The characteristics that can be exhibited by combining different bands also have great differences. Therefore, it is especially important to choose which combination method. By summarizing the experience of the predecessors, selecting the best visual effect, and the principle of comprehensive optimization, the 321-band true color synthesis is selected, and the color of the ground objects is vivid, which is conducive to the display of the map and the visual interpretation.

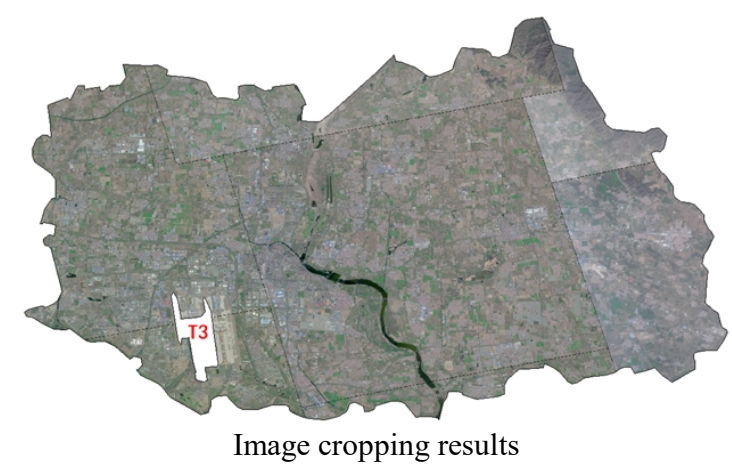

4.4 Remote Sensing Image Denoising

In this paper, a map containing a large amount of " salt and salt" noise is selected from the PMS data of Shunyi District remote sensing image to test the denoising effect of wavelet analysis and ICA combined algorithm proposed in this paper. And compared with the traditional wavelet transform denoising method, the results of the two denoising methods are evaluated based on the comparison of signal-to-noise ratio (SNR/d B) and root mean square error (RMSE). To verify the superiority of the proposed wavelet analysis and ICA algorithm in the actual remote sensing image processing compared with the traditional method, the original data selected in this experiment is a noisy full-color image, and the results are as follows:
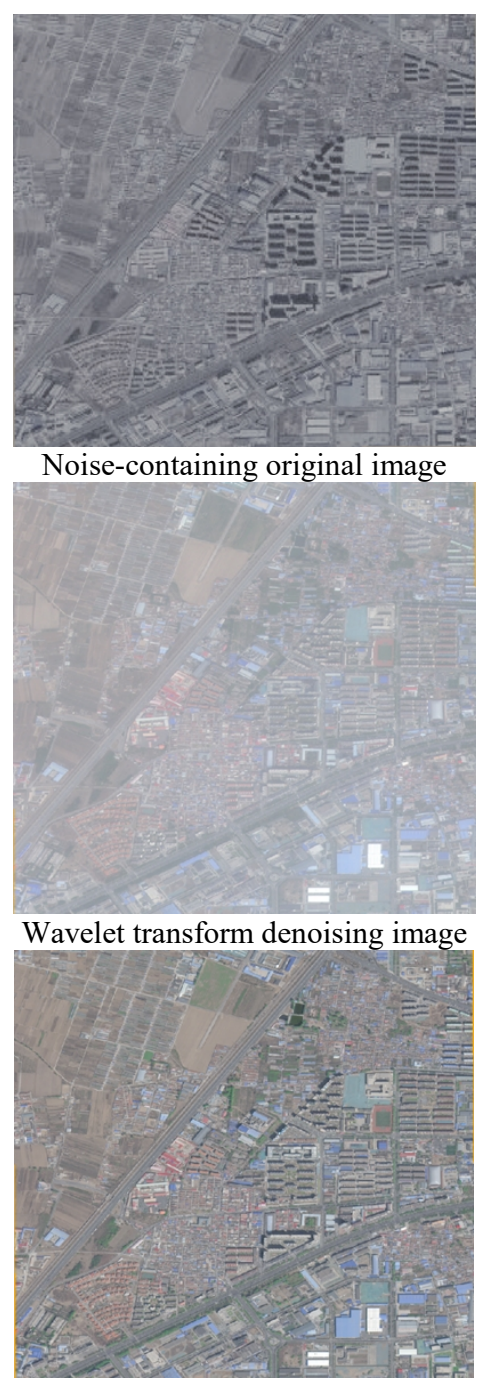

ICA-Wavelet Transform Denoising Image 
After comparing the signal-to-noise ratio $(\mathrm{SNR} / \mathrm{dB})$ and root mean square error (RMSE), it is found that the denoising effect of wavelet analysis and ICA combined denoising algorithm is better than the traditional wavelet transform algorithm. The feasibility of the ICA-wavelet denoising algorithm in the practical application of high-resolution remote sensing image denoising is proved.

\begin{tabular}{|l|l|l|l|}
\hline algorithm & Original image & $\begin{array}{l}\text { Traditional } \\
\text { wavelet }\end{array}$ & ICA-wavelet \\
\hline SNR/dB & 36.546 & 40.134 & 42.224 \\
\hline RMSE & 0.154 & 0.116 & 0.098 \\
\hline
\end{tabular}

\section{CONCLUSION}

By using the traditional wavelet transform denoising algorithm and the improved ICA-wavelet analysis denoising algorithm, respectively, the Shunyi District of Beijing after radiometric calibration, atmospheric correction, image fusion, image mosaic, image cropping and band pre-preparation were performed. The Beijing No. 2 PMS remote sensing image is denoised, and the signal-to-noise ratio $(\mathrm{SNR} / \mathrm{dB})$ and mean square error (RMSE) of the remote sensing image obtained by analyzing the two denoising methods are analyzed. The ICA-wavelet denoising algorithm is in the high score. The image processing results are superior to the traditional traditional wavelet transform denoising algorithm.

Remote sensing images inevitably introduce noise in the process of generation and transmission. These noises will greatly affect the quality of remote sensing images and increase the difficulty of analysis and processing of remote sensing images. High-resolution images have high cost and relatively large width, which is of great significance for noise reduction processing of high-resolution remote sensing images. Therefore, the necessary denoising processing should be carried out before applying the remote sensing image. When denoising or filtering the high-resolution remote sensing image, the useful information contained in the original remote sensing image should be preserved as much as possible and the noise in the image should be filtered out.

\section{REFERENCES}

Chang G, Yu B. 2000. Adaptive Wavelet Thresholding for Image Deno-ising and Compression[J]. IEEE Trans. on Image Proc, 9 (09).

Rafael C. Gonzalez, Richard E. Woods. 2015. Digital Image Processing Using MATLAB[M]. BEIJING: Publishing House of Electronics Industry.

Qu Ji-shuang,Wang Chao. 2012. A wavelet package-based data fusion method for multi. Temporal remote sensing image processing $[\mathrm{J}]$. Center for remote imaging sensing and processing (CRISP).

Rahimzadeh-Bajgiran P,Weiskittel A,Kneeshaw D,et al. Forests 2018.

Niemann K O,Quinn G,Stephen R,et al. Canadian Journal of Remote Sensing . 2015.
Tensor completion using total variation and low-rank matrix factorization[J] . Teng-Yu Ji,Ting-Zhu Huang,Xi-Le Zhao,TianHui Ma,Gang Liu. Information Sciences . 2016.

赵蓄, 刘淑英, 李红. 2013. 基于 ICA 的遥感图像的色彩分 类方法 $[\mathrm{J}]$. 航空计算技术.（06）.

张艺. 2010. ICA 技术及其在图像去噪中的应用 [D]. 东北石油 大学.

易尧华, 余长慧, 秦前清, 龚健雅. 2015. 基于独立分量分析 的遥感影像非监督分类 $[\mathrm{J}]$. 武汉大学学报 (信息科学版), 30 (01): 19-22.

韩子扬, 杜利明, 王金. 2016. 一种改进的小波阈值去噪方法 [J]. 现代电子技术, 39（17）:20-22. 